\begin{tabular}{|l|l|} 
REVISTA Revista Educación \\
ISSN: 0379-7082 \\
ISSN: 2215-2644 \\
revedu@gmail.com \\
Universidad de Costa Rica \\
Costa Rica
\end{tabular}

\title{
Educación universitaria en tiempos de pandemia: el caso del estudiantado de francés
}

Chao Chao, Kuok Wa; Cambronero Artavia, María del Pilar; Chacón Quesada, María de la Paz Educación universitaria en tiempos de pandemia: el caso del estudiantado de francés

Revista Educación, vol. 46, núm. 1, 2022

Universidad de Costa Rica, Costa Rica

Disponible en: https://www.redalyc.org/articulo.oa?id=44068165034

DOl: https://doi.org/10.15517/revedu.v46i1.45343

\section{(c) (1) 90}

Esta obra está bajo una Licencia Creative Commons Atribución-NoComercial-SinDerivar 3.0 Internacional. 


\section{Educación universitaria en tiempos de pandemia: el caso del estudiantado de francés}

French-Language University Students and Online Education during the Pandemic

Kuok Wa Chao Chao

Universidad de Costa Rica, Costa Rica

kuok.chao@ucr.ac.cr

DOI: https://doi.org/10.15517/revedu.v46i1.45343 Redalyc: https://www.redalyc.org/articulo.oa? $\mathrm{id}=44068165034$

(D) https://orcid.org/0000-0003-0683-1481

Maria del Pilar Cambronero Artavia

Universidad de Costa Rica, Costa Rica

maria.cambronero@ucr.ac.cr

(iD https://orcid.org/0000-0001-6894-5957

María de la Paz Chacón Quesada

Universidad de Costa Rica, Costa Rica

mariadelapaz.chacon@ucr.ac.cr

(D) https://orcid.org/0000-0003-2310-6169

Recepción: 25 Enero 2021

Aprobación: 18 Mayo 2021

\section{RESUMEN:}

En el año 2020, hubo grandes cambios en la enseñanza de una lengua debido a la pandemia global del COVID-19. Esta investigación busca describir las experiencias del estudiantado de francés como lengua extranjera en cuanto a la virtualidad de sus cursos. Se trata de una investigación de enfoque mixto, cuyo instrumento de recolección de datos fue un cuestionario compuesto de preguntas abiertas y cerradas aplicado a 101 participantes. Se realizó un análisis de contenido para las preguntas abiertas y un análisis estadístico para las preguntas cerradas. Como principales resultados, las nuevas prácticas de docentes desencadenaron resultados como el aumento en la creatividad, la cooperación y un rol más activo de la persona estudiante, así como la fatiga, el estrés y la sobrecarga. Gracias a los insumos obtenidos, se concluye que, en el caso del estudiantado de francés, es posible adaptar y llevar a cabo la mayoría de los cursos de la carrera.

Palabras Clave: Francés, Pandemia, COVID-19, Coronavirus, Virtualidad, TIC, Universidad.

\section{Abstract:}

In 2020, foreign language teaching experienced many changes as a result of the COVID-19 global pandemic. This paper aims to describe the French as a foreign language students' experiences in switching their language courses to an online format. This was a mixed-methods study that collected data using both closed and open-ended questions answered by 101 participants. Content analysis was executed for open-ended questions with statistical analysis for closed-ended questions. Noteworthy results include increased creativity, cooperation and even a more active role from students. However, students also experienced screen fatigue, stress and excessive workloads. It was concluded that remote classe satisfatory information provided, it can be concluded that it is possible to successfully adapt and conduct most courses remotely.

KEYwORDS: French, Pandemic, COVID-19, Coronavirus, Online Classes, ICT, University.

\section{INTRODUCCIÓN}

Desde el año 2020, el mundo está viviendo la crisis de salud más grande de estos últimos decenios: una enfermedad causada por un coronavirus, que brotó en Wuhan (China) en diciembre de 2019, se propagó rápidamente a muchos países y, según la Organización Mundial de la Salud (2020), evolucionó a ser pandemia global. Ningún país estaba preparado para esta emergencia médica, lo que implicó grandes cambios en 
diferentes aspectos de la sociedad. Este virus cambió la manera de relacionarse entre las personas, ya que se debe utilizar cubrebocas, mantener un distanciamiento social, lavarse las manos, entre otros aspectos.

Frente a esta nueva realidad, el sistema educativo, en general, ha tenido que adaptarse a estos cambios. De esta manera, muchas instituciones educativas, desde la educación primaria hasta la universitaria, cerraron sus puertas para implementar un nuevo modelo de enseñanza en el que se aprovecha el uso de herramientas tecnológicas con el fin de seguir impartiendo lecciones.

En el caso de Costa Rica, desde el mes de marzo, las instituciones educativas pasaron de la presencialidad a una educación remota, como lo es la Universidad de Costa Rica (UCR). El Centro de Coordinación Institucional de Operaciones (CCIO) de la UCR instruyó a la comunidad educativa, por medio del Comunicado \#3, sobre los acuerdos de solicitar al personal docente suspender, a partir de este momento, las clases presenciales e implementar una modalidad de enseñanza virtual que garantice la continuidady calidad, asi como implementar de forma inmediata el trabajo remoto para el personal administrativo. Esta decisión, tomada en la semana del inicio del curso lectivo 2020, con la intención de salvaguardar la salud de toda la comunidad universitaria, traería consecuencias insospechadas para la Institución, que no radicaban únicamente en la modalidad del curso lectivo. Así como el contexto de la pandemia afectó la manera en que se impartían los cursos, la llegada de las tecnologías en la sociedad actual en el proceso educativo está produciendo cambios importantes en la metodología (Martínez, 2017).

Con la iniciativa de la UCR por impartir los cursos presenciales de manera virtual, el apoyo más grande para personas docentes y estudiantes ha sido la Unidad de Apoyo a la Docencia Mediada con TICS (METICS). Como resultado de la evolución epidemiológica del COVID-19 en Costa Rica, en el Comunicado \#3 del 11 de marzo de 2020 se hizo la solicitud al cuerpo docente de suspender a partir de ese momento las clases presenciales e implementar clases bajo una modalidad virtual. De esa manera se impulsó inmediatamente el trabajo remoto para cargos docentes y administrativos, salvo los casos que por su modalidad no pudieran ser virtuales y debieran ser analizados por la Dirección de Escuela o Facultad. Además, se habilitó un sitio internet en el que se pone a disposición de la comunidad universitaria información actualizada sobre el contexto actual y los comunicados emitidos.

La UCR, en un esfuerzo por proveer a personas docentes y estudiantes de herramientas, puso a disposición otras plataformas como Zoom y Mconf para llevar a cabo videoconferencias. Por otra parte, en coordinación con el Instituto Costarricense de Electricidad (ICE) se realizaron acciones para duplicar el ancho de banda de Internet en todas las sedes y recintos de la institución, además de lograr la eliminación del cobro por consumo de datos móviles en el dominio ucr.ac.cr. De esta manera, la universidad implementó durante 2020 la virtualidad en los cursos.

Bachelor (2019) refiere a la virtualidad en los cursos como el uso de tecnologías, de software de aprendizaje y de Internet en un entorno $100 \%$ en línea sin ninguna presencia física. Las tecnologías de la información y de la comunicación (TICS) son un grupo especializado de programas y herramientas (Internet, televisión, radio, celular, entre otros) que han facilitado desde hace tiempo el acceso a recursos pedagógicos, a la producción, a la difusión de información, a la interacción y colaboración entre el profesorado y el estudiantado (Gil, 2019; Soto, 2018).

Las TICS han estado presentes desde finales de la Segunda Guerra Mundial con el Método Audio-Oral (MAO) y luego, en los años 60, con el método Estructura Global Audiovisual (SGAV), se diversificó el uso de estas herramientas para la enseñanza de idiomas extranjeros (Beauné, 2011). Al-Assaf (2008) afirma que la virtualidad que utiliza las TICS puede motivar al estudiantado en su aprendizaje; sin embargo, el gran inconveniente es el costo del equipo, ya que no mucha gente dispone de recursos suficientes para la compra de una computadora. Hocine (2011) agrega que otro de los inconvenientes es la falta de capacitación del personal docente en su utilización.

Ait-Dahmane (2011) menciona que las TICS proveen medios innovadores para la difusión de los conocimientos y para la exploración de estrategias de aprendizaje que favorecen la construcción de las 
competencias, tales como la accesibilidad de la información, la interactividad, los intercambios de correos y de comunicación. Hocine (2011) encuentra también que las TICS favorecen la flexibilidad y el acceso de una gran gama de recursos.

Durante el contexto de COVID-19, el uso generalizado de las TICS para la enseñanza de una lengua extranjera ha sido la manera utilizada para seguir los cursos. Kem-Mekah (2020) realizó una investigación sobre el contexto camerunés y encontró que el cuerpo docente del departamento de lenguas extranjeras empleó, sobre todo, la aplicación de WhatsApp para adaptar los cursos a la virtualidad. De esta manera, las clases fueron impartidas desde esta aplicación y se utilizó para corregir o hacer ejercicios, cantar, debatir, recitar fragmentos, explicar, aclarar dudas, etc. También, encontró que el personal docente empleó otras aplicaciones como Facebook, Twitter, Instagram o Google Classroom. Al utilizar estas aplicaciones, la persona autora evidenció un cambio de paradigma, ya que pasó de un modelo lineal de aprendizaje, en el cual el profesorado era el centro de todo el proceso, a uno dinámico, en el cual el estudiantado adquirió un papel más activo en su proceso de aprendizaje. En su investigación de tipo descriptivo con un enfoque filosófico mixto con 39 docentes de diversas disciplinas a través de un cuestionario de tipo dicotómico, Navarro-Hudiel (2020) identificó que las herramientas más utilizadas por el personal docente para sus clases son: Zoom, Microsoft Teams, Google Meat, Youtube.

En su investigación con docentes de la Universidad de Valladolid, Sánchez (2020) investigó sobre la adaptación del profesorado durante el contexto de la pandemia para su curso de inglés. Encontró que la creatividad del personal docente fue lo más importante durante la adaptación en este contexto y que el profesorado ha tenido que atender al alumnado de la mejor manera posible para asegurar la calidad de su formación, por lo que debió ser flexible para adecuarse a este modelo, con el esfuerzo que ello conlleva para las personas profesoras y alumnas.

En una investigación documental, Pastran et al. (2020) estudiaron los beneficios de las TICS en la enseñanza de lenguas extranjeras. Las personas autoras coincidieron con los resultados obtenidos por Sánchez (2020) y agregaron que las TICS permitían al personal docente ofrecer un acompañamiento al estudiantado durante su proceso de aprendizaje, especialmente en la distancia, puesto que el estudiantado podía acceder a distintas actividades interactivas con personas docentes y otros estudiantes, así desarrollarían competencias comunicativas como la producción y la comprensión de texto, la expresión oral, entre otras. Además de las ventajas identificadas, encontraron otras como el bajo costo, la flexibilidad horaria, el fomento de la creatividad y de la calidad de los materiales didácticos.

En una investigación con metodología mixta a través de un cuestionario enviado a 400 estudiantes y 100 docentes, Rechidi et al. (2020) encontraron que el uso de las TICS permitía atraer más estudiantes a los cursos y seguir la continuidad de las clases en este tipo de contexto; sin embargo, los mayores inconvenientes fueron el acceso a Internet o los problemas de conexión en Marruecos, la falta de acompañamiento del profesorado, la duración de la clase que se limitaba a 40 minutos por Zoom, la falta de contacto y de interacción con la persona docente o con sus pares.

En su investigación cuantitativa a 200 estudiantes de la Universidad Técnica de Manabi, Cevallos et al. (2020) investigaron sobre la metodología de las clases virtuales del inglés como lengua extranjera durante el contexto de la pandemia a través de una investigación cuantitativa. Las personas autoras encontraron que la herramienta tecnológica Moodle favorecía el aprendizaje del inglés a través de actividades lúdicas. Las aulas virtuales permitían emplear todo tipo de conocimiento, técnica o actividad, solamente requería el dominio de las herramientas tecnológicas, además de permitir la relación de espacios tecnológicos con innovación educativa, análisis de actividades sobre los contenidos, participación activa y creativa del estudiantado, que potenciaba el aprendizaje, la cooperación y la creación de nuevas iniciativas con resultados altamente positivos.

Por último, en cuanto a las investigaciones sobre la percepción del estudiantado en lenguas extranjeras sobre la virtualidad en cursos de lengua, se identificó una investigación cuantitativa de Maican y 
Cocorada (2021) con 207 estudiantes. Las personas autoras encontraron como resultados principales que el estudiantado percibió una flexibilidad en el proceso de aprendizaje, ya que el material estaba disponible por largo tiempo en la plataforma. Sin embargo, el estudiantado consideró una disminución en cuanto a la atención individualizada del profesorado a través de la retroalimentación. Además, otras dificultades identificadas eran la falta de preparación en el uso de herramientas tecnológicas por parte profesorado, los problemas de conexión a Internet, la ausencia de herramientas tecnológicas en su hogar, como micrófono o cámaras. Otros inconvenientes encontrados fueron las emociones negativas generadas por este tipo de aprendizaje debido a la falta de interacción con sus pares o con el profesorado.

Como síntesis, el uso de herramientas tecnológicas ha estado presente en la enseñanza de una lengua extranjera. Además, durante el contexto del COVID-19, las instituciones educativas han recurrido al uso de la tecnología para continuar sus procesos educativos. En el caso concreto de una lengua extranjera, se observa que las investigaciones giran en torno al inglés y a la percepción del personal docente sobre la adaptación a la virtualidad. Algunas tratan del caso del estudiantado del inglés en cuanto a la virtualidad. Sin embargo, casi ninguna trata el caso del francés y de la percepción del estudiantado universitario. Por lo tanto, esta investigación busca enriquecer esta temática, al aportar un análisis sobre las percepciones que tiene la población estudiantil sobre la adaptación del aprendizaje del francés como lengua extranjera a la virtualidad. De esta manera, se plantean la siguiente pregunta de investigación general y las preguntas específicas de investigación:

\section{Pregunta general.}

¿Cuáles son las percepciones de la población estudiantil sobre la adaptación del aprendizaje del francés como lengua extranjera a la virtualidad?

\section{Preguntas específicas de investigación}

¿Cuáles son las ventajas y desventajas del aprendizaje de una lengua extranjera en modalidad virtual durante el contexto de la pandemia del COVID-19?

¿Cuál fue la experiencia del estudiantado sobre los cursos de francés durante el contexto de la pandemia del COVID-19?

¿Cómo percibe el estudiantado la preparación y adaptación del personal docente a la virtualidad durante el contexto de la pandemia del COVID-19?

Este artículo tendrá la siguiente estructura. Primeramente, se presentará el concepto de TICS, así como sus ventajas y desventajas, para finalizar con la virtualidad en el proceso educativo. Luego, se explicará el procedimiento metodológico utilizado, así como el enfoque, la técnica, las características del contexto y de las personas participantes, y la manera de analizar los datos. Posteriormente, se mostrarán los resultados según las preguntas de investigación. Por último, se discutirá sobre los resultados obtenidos, así como las conclusiones.

\section{LAS TICS}

En cuanto a las TICS, la UNESCO señala que estas pueden complementar, enriquecer y transformar la educación. Asimismo, comparte conocimientos relacionados a las formas en que la tecnología facilita el acceso universal a la educación, reduce las diferencias en el aprendizaje, apoya el desarrollo del profesorado, así como mejora la calidad y la pertinencia del aprendizaje.

Las TICS son un conjunto de herramientas cuyas funciones son el procesamiento, el almacenamiento, la sintetización, la representación y la transmisión de todo tipo de información, que apoyan el proceso de enseñanza-aprendizaje de una lengua y que permiten a las personas que se encuentran en diferentes espacios y momentos comunicarse e interactuar (Gil, 2019; Lizasoain et al. 2018; Simons, 2010; Soto, 2018).

En su trabajo sobre el quehacer docente, Ruiz y Aguirre (2013) se refieren a la función de las TICS:

Con las TICS (antes llamadas nuevas tecnologías) se crean espacios de enseñanza y aprendizaje no sólo en un aula convencional, aquella donde los estudiantes y el profesor se encuentran en el mismo tiempo y espacio, sino que se generan 
espacios virtuales donde, además de intercambiar información, se dan relaciones mediáticas, de formación, interacción, trabajo, colaboración e investigación (p.109).

Martínez et al. (2014) señalan que, cuando se trata de fenómenos de innovación educativa ligados a la tecnología, se da una constante hibridación entre diversos dispositivos técnicos, lenguajes, programaciones y sistemas digitales que dan lugar a nuevos métodos y sistemas. Por otra parte, las personas autoras mencionan que, cuando se ponen en práctica en el ámbito educativo, se seleccionan aquellas a su alcance y las que sean más coherentes y adaptables. De esta manera, según Martínez et al. (2014), la innovación educativa gira en torno a la emergencia y la consolidación de diversos marcos y modelos de relación entre las personas participantes en esos procesos, con el fin de establecer relaciones mediadas por dispositivos y procedimientos tecnológicos.

Moreira-Segura y Delgadillo-Espinoza (2015) afirman que, en lo referente a las nuevas tecnologías de información y comunicación (NTIC), se destacan como facilidades de la virtualización, el rompimiento de la barrera de la distancia, la rigidez de los horarios y la facilidad de la distribución del tiempo de estudio, además de la posibilidad de combinarla con las múltiples ocupaciones que conlleva el estilo de vida moderna. Por estas y otras razones, afirman que en los procesos virtuales debe prevalecer la comunicación fluida y efectiva, en una atmósfera cálida y con un acompañamiento constante de las actividades que realizan las personas participantes, con el fin de favorecer la cercanía y calidez entre los interlocutores.

Durán et al. (2015) destacan dentro de las características que diferencian la educación presencial de la virtual, que en esta última existe una mayor autonomía e independencia de parte del estudiantado para el desarrollo de su proceso de aprendizaje, pues cada estudiante trabaja a su propio ritmo, al mismo tiempo que se da una adquisición de competencias a través de este modelo.

Por su parte, Belloch (2012) enfatiza el hecho de que bajo la modalidad virtual el estudiantado necesita de igual manera ser apoyado y guiado por la persona docente, pues tener información disponible no garantiza la adquisición de conocimientos. Es por ello que la autora insiste en que el estudiantado debe aprender a aprender, acceder a la información, comprenderla, resaltar ideas fundamentales, estructurarla y tener una visión crítica sobre esta; el estudiantado construye su conocimiento bajo la guía de la persona docente; las TICS, en este panorama, son elementos adecuados para la creación de entornos que apoyen el aprendizaje constructivo, colaborativo y por descubrimiento.

Asimismo, Belloch (2012) destaca como ventajas de las TICS la información variada, la flexibilidad instruccional, la complementariedad de códigos, el aumento de la motivación, las actividades colaborativas y la potencialización de la innovación educativa. En cuanto a las desventajas destaca la existencia de la pseudoinformación, la sobrecarga de información y la dependencia tecnológica.

Además de lo mencionado por Belloch (2012), las TICS presentan las siguientes ventajas para el aprendizaje de un segundo idioma (Burbat, 2016; Chao, 2014; Contreras, 2008; Gavarri, 2016; Rodríguez, 2016):

a) Fomenta el proceso individual y autónomo de aprendizaje

b) Permite un acceso no lineal de la información

c) Ayuda a superar las limitaciones temporales y geográficas entre el profesorado y el estudiantado

d) Se da un acceso rápido a la información

e) Fomenta el trabajo cooperativo entre las personas participantes

En cuanto a sus desventajas, se encuentran las siguientes (Chao, 2014; Contreras, 2008):

a) La dificultad de acceso y de uso

b) El exceso de libertad para el estudiantado

c) La generación de ansiedad por el contacto continuo con la computadora

Con respecto a la virtualidad en el proceso educativo, Durán (2015) explica cómo las TICS no son solo un medio para ello, sino que su uso construye un nuevo espacio educativo. Este espacio es conocido 
como educación virtual, de carácter flexible, donde las personas estudiantes deben compatibilizar su actividad laboral y familiar con su formación, gracias a la posibilidad de desarrollarla en su propio domicilio.

Según la misma persona autora, la diferencia entre la educación virtual y la presencial radica en la existencia de una mayor autonomía e independencia en el proceso de aprendizaje. Es decir, el estudiantado universitario establece su propio ritmo de trabajo y percibe sus objetivos de aprendizaje de una manera más práctica, ya que este tipo de estudiante, con frecuencia, desarrolla una actividad laboral vinculada a sus estudios formales. Ese valor práctico de la formación favorece ampliamente su motivación intrínseca.

De acuerdo con Durán (2015), la educación virtual exige al estudiantado una mayor responsabilidad, compromiso, autonomía y autorregulación durante su proceso de aprendizaje. Además, este tipo de educación busca también que el estudiantado establezca relaciones y situaciones de aprendizaje compartido, cooperativo y colaborativo con otras personas participantes para enriquecer sus experiencias cognitivas (Durán, 2015).

Las personas autoras, Moreira-Segura y Delgadillo-Espinoza (2015), agregan, además, que un entorno virtual requiere un diseño pedagógico donde se privilegien:

La flexibilidad y la capacidad de adaptación, el saber aprender a aprender, la habilidad de navegar entre mares de información reconociendo lo que es confiable y útil, la percepción de sí mismo como un generador de conocimientos y no solamente como un consumidor de estos, la disposición a trabajar colectivamente en la resolución de un problema (p.125).

Las personas autoras aseguran, también, que un curso en modalidad virtual no solo requiere un ajuste en los contenidos, la evaluación o los roles de participantes y personas tutoras, además, demanda un pensamiento flexible y holístico por parte de quienes comparten esta modalidad de experiencia educativa.

Implementar la virtualidad en los procesos de enseñanza-aprendizaje puede brindar réditos productivos, siempre que se realice desde una posición proactiva y flexible, donde se comprenda que la persona docente es tutora, que forma parte del proceso, pero no es su protagonista. En este contexto, el cuerpo académico universitario tiene la posibilidad de experimentar nuevas modalidades, al innovar en las actividades que propone y con ello colaborar en la superación del entorno educativo (Moreira-Segura y Delgadillo-Espinoza, 2015).

\section{Metodología}

\section{Tipo de investigación}

Es una investigación que se sitúa en el paradigma pragmático que considera que el conocimiento se desarrolla a través de la acción y que es una consecuencia de la investigación, como es el caso del enfoque mixto (Fortin, 2010). Desde ese paradigma, se emplean múltiples puntos de vista para resolver un problema de investigación (Fortin, 2010). El diseño de la investigación se ubica en un enfoque mixto de tipo exploratorio que busca comprender en profundidad un fenómeno complejo (Creswell, 2009; Fortin, 2010; Pereira, 2011).

\section{Contexto de la universidad y la carrera}

La investigación se realizó en la Universidad de Costa Rica, específicamente en la carrera de Bachillerato en Francés y Bachillerato en la Enseñanza del Francés. Las dos carreras tienen una duración de cuatro años. Los cursos de lengua, como expresión escrita, expresión oral, gramática, fonética, literatura y cultura son matriculados por estudiantes de ambas carreras. En el caso del Bachillerato en Francés, el estudiantado tiene que matricular a un bloque optativo de 16 créditos y puede escoger tres opciones: inglés, turismo o traducción. 
En el caso del Bachillerato en la Enseñanza del Francés, el estudiantado lleva los cursos que corresponde la parte de didáctica, pedagogía, evaluación, etc.

\section{Participantes}

La muestra de la población participante es no probabilística por conveniencia, lo que permite seleccionar aquellos casos accesibles que acepten ser incluidos y que respondan a los criterios de inclusión (Fortin, 2010; Otzen y Manterola, 2017). En el caso de esta investigación, los criterios de inclusión son: ser estudiantes de las carreras antes mencionadas, haber matriculado un curso de estas carreras en el primer semestre de 2020 y llevar el curso en el contexto de la pandemia. Por lo tanto, las personas participantes están compuestas por estudiantes de ambas carreras. En la Tabla 1, se detallan sus características:

TABLA 1

Características de las personas participantes.

\begin{tabular}{ll}
\hline Características & Datos \\
\hline Sexo & $\mathrm{N}=34$ (hombres) $\mathrm{N}=67$ (mujeres) \\
Edad & $\mathrm{N}=41$ (17 a 20 años) $\mathrm{N}=46$ (21 a 25 años) $\mathrm{N}=8$ (26 a 30 años) $\mathrm{N}=6$ (31 o más) \\
Carrera empadronada & $\mathrm{N}=70$ (Bachillerato en Francés) $\mathrm{N}=36$ (Bachillerato en Enseñanza del Francés) \\
Año de la carrera & $\mathrm{N}=26$ (Primer año) $\mathrm{N}=33$ (Segundo año) $\mathrm{N}=20$ (Tercer año) $\mathrm{N}=22$ (Cuarto año) \\
Matriculada en bloque completo & $\mathrm{N}=57$ (Sí) $\mathrm{N}=44$ (no) \\
\hline
\end{tabular}

Fuente: elaboración propia.

\section{Instrumentos de recolección de datos}

En aras de conocer la percepción del estudiantado sobre los cursos virtuales durante el primer semestre de 2020, la Comisión de Autoevaluación y Gestión de la Calidad del Bachillerato en Francés se dio a la tarea de confeccionar un cuestionario para la población estudiantil de primer a cuarto año de carrera. El enlace del cuestionario se compartió por correo al profesorado para que lo hiciera llegar, ya fuera por correo electrónico o por medio de la plataforma Mediación Virtual, a las personas estudiantes. Adicionalmente, dicho enlace se publicó en la página de Facebook de la carrera.

Para recolectar los datos se utilizó un cuestionario que fue aplicado en línea durante el mes de junio, julio y agosto de 2020. El cuestionario se realizó con la aplicación de Google forms. El consentimiento se incluyó en la primera página del cuestionario y se le informó al estudiantado que la duración para responderlo era de 10 a 20 minutos. El cuestionario contiene un total de 24 preguntas, de las cuales cinco corresponden a la información personal de las personas participantes, doce son sobre su percepción sobre los cursos en la virtualidad y siete son sobre el uso de las plataformas virtuales para informarse. El cuestionario contenía nueve preguntas abiertas y quince preguntas cerradas, en las cuales el estudiantado escogía una de las dos opciones o el nombre del curso.

Las preguntas cerradas fueron utilizadas para recolectar algunas informaciones personales del estudiantado sobre el tipo de herramienta usada para informarse y sobre el tipo de curso que se adapta a la modalidad virtual, bimodal o presencial. No se utilizó ningún tipo de escala Likert para las preguntas cerradas, ya que las respuestas eran solamente sí. no. Por lo tanto, se empleó una escala dicotómica. En cambio, la mayoría de las preguntas abiertas se emplearon para conocer la percepción del estudiantado sobre los cursos en la virtualidad. 
El cuestionario fue revisado por tres personas especialistas y se pidió a una persona estudiante realizarla antes de la aplicación a las personas participantes, esto para saber si el instrumento permitía recolectar la información deseada y si las preguntas tendrían claridad para las personas participantes. Se realizaron varias modificaciones de redacción de las preguntas abiertas y se agregaron cuatro preguntas más antes de su aplicación definitiva.

En este artículo no se presentará el tipo de herramienta tecnológica usada para informarse, ya que esta información será utilizada para otro artículo.

\section{Procedimiento de análisis de datos}

Para las preguntas abiertas se realizó un análisis de contenido. El análisis de contenido de las preguntas abiertas se hizo a partir de la codificación con la identificación de palabras clave y segmentos, en busca de los patrones recurrentes, de manera que se categorizaron en las siguientes categorías: experiencia, ventajas, inconvenientes y preparación del profesorado. Luego, cada categoría se dividía en subcategorías, por ejemplo: la categoría de ventajas se separaba en flexibilidad, comodidad, disponibilidad de materiales, accesibilidad de recursos, entre otras. Estas categorías y subcategorías fueron establecidas previamente.

Para las preguntas cerradas sobre los cursos que pueden ser ofrecidos de manera virtual, bimodal y presencial, se realizó un análisis estadístico porcentual de los resultados por medio de la herramienta de Excel, en donde se indica la frecuencia de respuesta para cada curso propuesto.

Por último, para la validación del análisis de contenido, se realizó un contracodaje de los datos. Cada una de las personas investigadoras realizó la clasificación de los datos según las categorías y las subcategorías establecidas previamente. Luego, se comparó la clasificación para validar el análisis de contenido y se obtuvo un 95,6\% de coincidencia. Los casos en donde no hubo coincidencias se analizaron y se discutieron para buscar la categoría o la subcategoría más acorde con los datos.

\section{Presentación de resultados}

En esta sección se presentan los resultados obtenidos en la investigación. Cabe mencionar que la primera pregunta de la investigación busca identificar las ventajas y las desventajas del aprendizaje del francés en modalidad virtual durante el contexto de la pandemia del COVID-19.

El estudiantado considera las siguientes ventajas del aprendizaje del francés en modalidad virtual durante la pandemia del COVID-19: el ahorro de tiempo de traslado en bus (33,6\%), la flexibilidad para la realización de los trabajos o su entrega (15,8\%), la disponibilidad de los materiales del curso (15,8\%), el ahorro de dinero (11,8 \%), la comodidad de recibir clases en la casa (9,9\%), el evitar salir de la casa $(8,9 \%)$, la organización de su propio ritmo de trabajo y de su aprendizaje (8,9\%), el provecho del tiempo libre $(7,9 \%)$, la generación de menos estrés $(6,9 \%)$, la autonomía del estudiantado para el aprendizaje $(4,9 \%)$, la combinación de trabajo y estudio $(3,9 \%)$ y la accesibilidad a una variedad de recursos $(2,9 \%)$ como se ilustra en la Figura 1. 


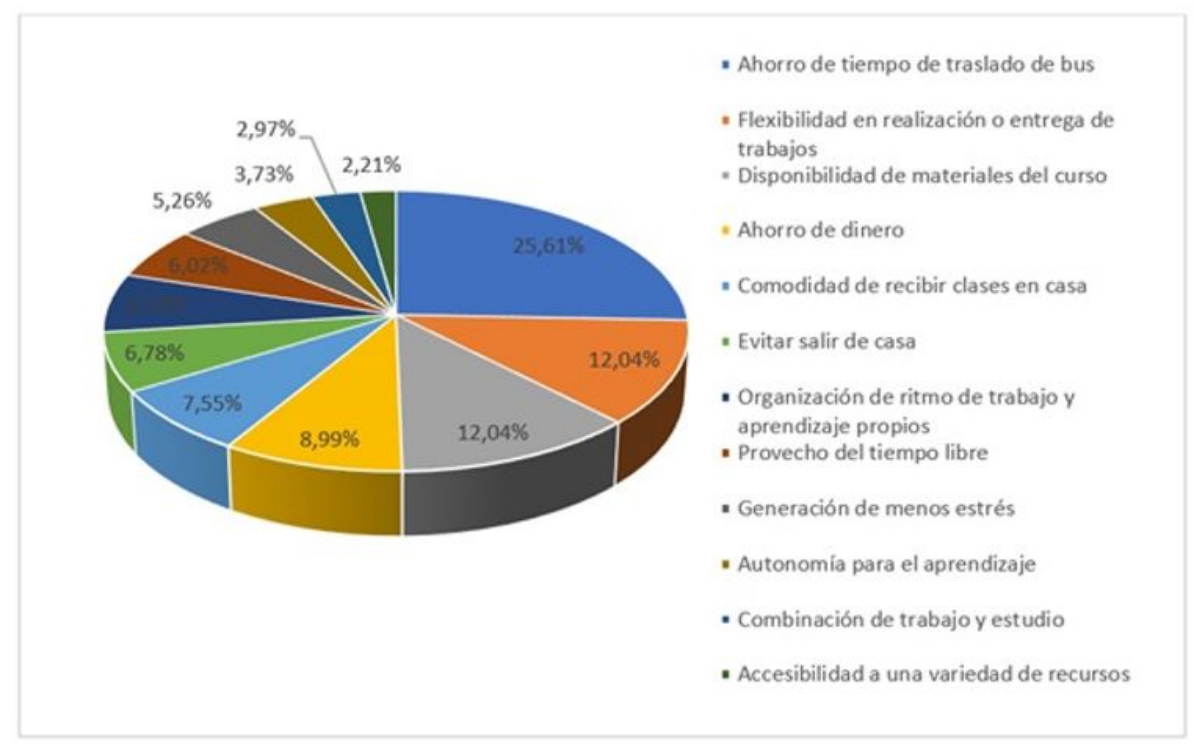

FIGURA 1.

Ventajas del aprendizaje del francés en modalidad virtual.

Fuente: elaboración propia.

Además, existen otras ventajas que fueron mencionadas, cada una, por una sola persona estudiante, por ejemplo: el aumento de la responsabilidad del personal docente (sujeto 57, 2 de julio de 2020), la facilidad de los trabajos y de las actividades evaluativas (sujeto 37, 30 de junio de 2020), la efectividad de la comunicación entre personal académico y estudiantado, y una nueva forma de evaluación (sujeto 04, 28 de junio de 2020).

Así como se han resaltado aspectos positivos de esa modalidad, el estudiantado menciona también la existencia de inconvenientes y de dificultades con esta nueva modalidad de aprendizaje del francés, por ejemplo: los problemas de conexión a Internet $(45,5 \%)$, la ausencia de interacción con el profesorado y con sus pares $(18,8 \%)$, una mayor carga académica (15,8\%), la dificultad para evacuar dudas de forma inmediata $(15,8 \%)$, la poca preparación del profesorado a la virtualidad (9,9\%), la inexistencia de una corrección inmediata del profesorado (6,9\%), la dificultad de desarrollar la expresión oral en la virtualidad (6,9\%), la disminución de tiempo de clases sincrónicas (5,9\%), la dificultad para concentrarse (4,9\%), el cansancio de estar en frente de la computadora (3,9\%), el cansancio cuando la clase se extiende por más de una hora $(3,9$ $\%)$ y la falta de acompañamiento del profesorado (2,9\%), como se ilustra en la Figura 2. 


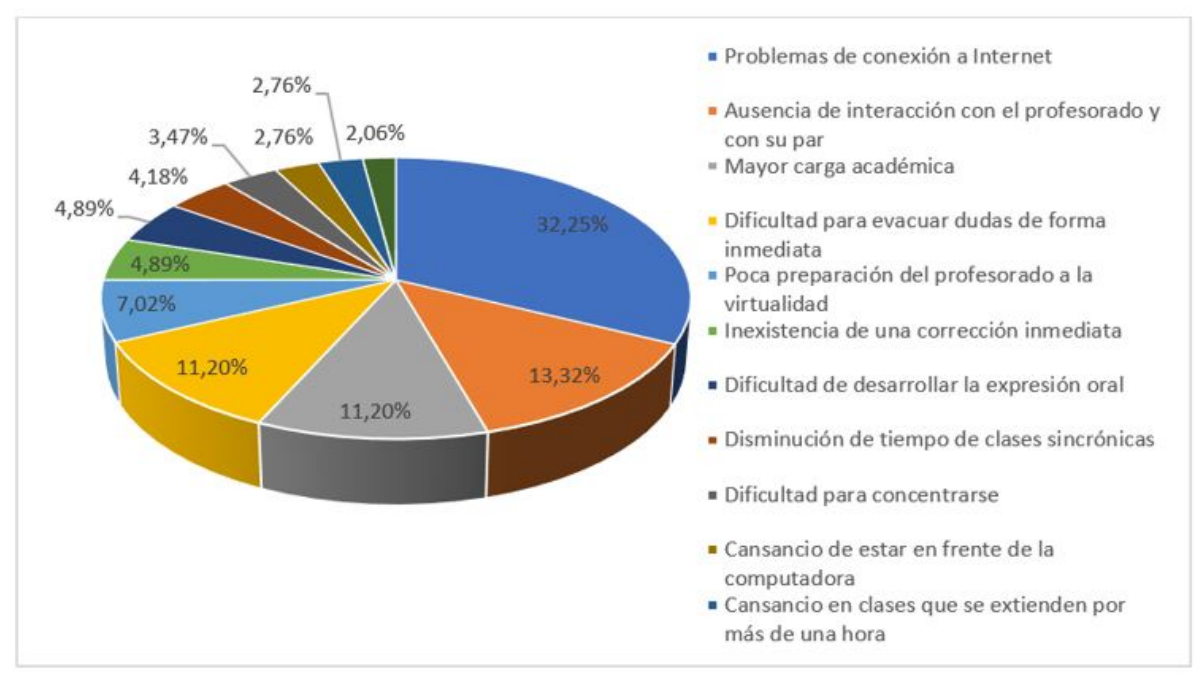

FIGURA 2.

Desventajas del aprendizaje del francés en modalidad virtual

Fuente: elaboración propia.

Además, existen otros inconvenientes que fueron mencionados por una sola persona estudiante: la falta de dinamismo (sujeto 57, 02 de julio de 2020), el fomento de la desigualdad del estudiantado (sujeto 17, 29 de junio de 2020), el fomento del sedentarismo (sujeto 85, 08 de julio de 2020), la falta de empatía del profesorado (sujeto 96, 31 de julio de 2020), el aumento de carga de trabajo en los trabajos en grupo (sujeto 10, 29 de junio de 2020), la irresponsabilidad del personal docente (sujeto 68, 05 de julio de 2020), la mala comprensión de las instrucciones (sujeto 06, 28 de junio de 2020).

En cuanto a la segunda pregunta de investigación, esta busca documentar las experiencias que ha tenido el estudiantado durante el aprendizaje del francés en el contexto de la pandemia del COVID-19 mediante preguntas abiertas, en las cuales pudieron expresar sus opiniones. El análisis de estos resultados se da a dos niveles: el primero, de tipo estadístico, cuantifica la incidencia de una temática en el total de respuestas recibidas, las cuales se exponen en este apartado; el segundo, de tipo cualitativo, destaca las vivencias en su contexto, las cuales se detallan en el apartado de análisis de resultados.

Para comenzar, la percepción del estudiantado en cuanto a si el profesorado estaba preparado para impartir cursos en modalidad virtual al inicio de la pandemia, se vio muy dividida. Está claro que existen diferencias entre cursos y entre cuerpo docente, y el estudiantado se mostró crítico al respecto.

A continuación, se presenta el resumen de la percepción del estudiantado respecto a si el personal docente se encuentra preparado para impartir los cursos según las variables de género, seguimiento completo del bloque lectivo y carrera de empadronamiento del estudiantado participante del estudio: 


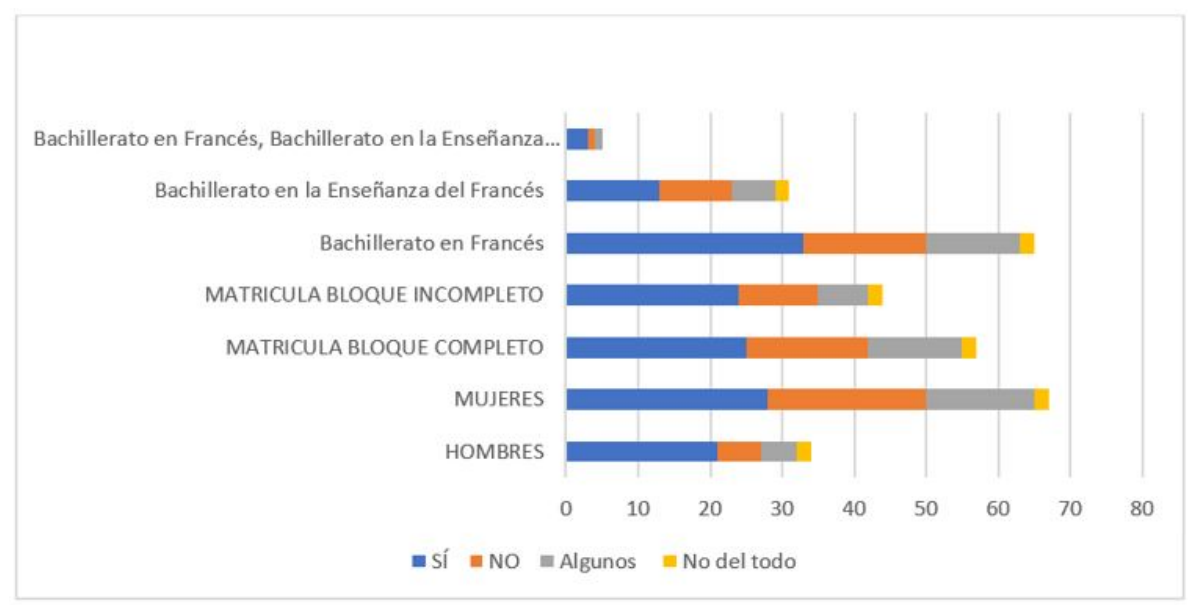

FIGURA 3.

Percepciones respecto a la preparación del personal docente para impartir los cursos en modalidad virtual. Fuente: elaboración propia.

La Figura 3 condensa la información obtenida, la cual es igualmente presentada en la tabla, la cual muestra, en términos numéricos, cuántas personas consideran que el cuerpo docente está preparado para asumir la virtualidad.

TABLA 2

Percepciones respecto a la preparación del personal docente para impartir los cursos en modalidad virtual por variable.

\begin{tabular}{|c|c|c|c|c|}
\hline \multirow[b]{2}{*}{ VARIABLE } & \multicolumn{4}{|c|}{ PREPARACIÓN DOCENTE } \\
\hline & Sí & No & ALGUNOS O ALGUNAS & NO DEL TODO \\
\hline \multicolumn{5}{|l|}{ Género } \\
\hline Hombres & 21 & 6 & 5 & 2 \\
\hline Mujeres & 28 & 22 & 15 & 2 \\
\hline \multicolumn{5}{|l|}{ Condición de matrícula } \\
\hline Matriculado en bloque completo & 25 & 17 & 13 & 2 \\
\hline Matriculado en bloque incompleto & 24 & 11 & 7 & 2 \\
\hline \multicolumn{5}{|l|}{ Carrera de empadronamiento } \\
\hline Bachillerato en Francés & 33 & 17 & 13 & 2 \\
\hline Bachillerato en la Enseñanza del Francés & 13 & 10 & 6 & 2 \\
\hline Bachillerato en Francés, Bachillerato en la Enseñanza del Francés & 3 & 1 & 1 & 0 \\
\hline
\end{tabular}

Fuente: elaboración propia.

La Tabla 2 muestra que, indistintamente de si las personas matriculan su bloque completo o no, quienes opinan que el cuerpo docente sí está preparado son prácticamente equivalentes. De igual manera, las 4 personas que opinan que el personal no está del todo preparado, dio respuestas idénticas, sin diferencia de género, condición de matrícula o carrera de empadronamiento. En cuanto a las respuestas que declaran que el cuerpo docente no está preparado o que algunas personas sí lo están pero algunas no, la relación es de 2 a 1 y hasta de 3 a 1, según la variable, pero se debe considerar que los números globales reflejan que respondieron más del doble de mujeres que de hombres, más del doble de estudiantes del Bachillerato en Francés que en Enseñanza del Francés, y un $30 \%$ más de personas estudiantes que matricula el bloque completo que aquellas que no lo hacen.

En vista de lo observado en la figura anterior, se determina realizar un análisis más específico según las respuestas proporcionadas en las preguntas abiertas, sin distinción de las variables supracitadas, las cuales se exponen en la Figura 4 (a continuación) y se explicarán con detalle posteriormente. 

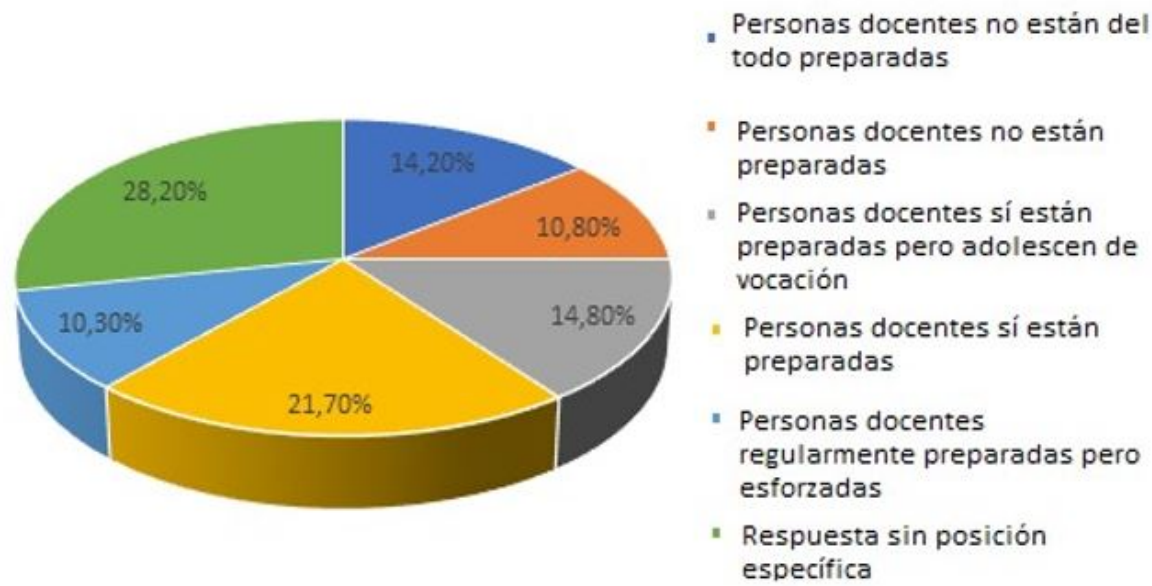

FIGURA 4.

Percepción sobre la preparación del personal docente en la virtualidad. Fuente: elaboración propia.

Una parte del estudiantado fue tajante en declarar que las personas docentes no estaban preparadas para asumir la virtualidad (10,8 \%), por ejemplo: "no, muchos profesores no supieron adecuar sus cursos correctamente, por lo que la opción que tomaron fue sobrecargar a los estudiantes con trabajos, tareas, exposiciones, etc." (sujeto 99, 05 de agosto del 2021); "no realmente, como dije muchos profes no están teniendo en cuenta las dificultades de hacer trabajos en grupo por ejemplo" (sujeto 10, 29 de junio del 2020); mientras que otra parte suavizó el balance al indicar que algunas personas docentes no lo están, o no del todo (14,20 \%), por ejemplo: "algunos no parecen funcionar bien en modalidad virtual, se les complica y no se adaptan del todo bien. Además, no todos mantienen el debido equilibrio entre el trabajo sincrónico y asincrónico" (sujeto 06, 28 de junio del 2020).

Entre los argumentos más definitivos se indica que el factor edad no ha sido determinante, como lo muestra este verbatim: "no y lo más curioso es que los más jóvenes juegan de que saben mucho y no pueden adecuar una clase virtualmente... cuando otros profes más viejos han podido exitosamente" (sujeto 101, 09 de agosto del 2021), pues aún las personas docentes jóvenes han fallado en adaptarse a la virtualidad, ya que los cursos se extienden por dos horas o más, lo que causa pérdida de interés en el estudiantado y, para compensar su falta de adaptación, sobrecargan al estudiantado con trabajos, tareas, exposiciones y otras actividades con la intención de solventar la falta de explicaciones y prácticas presenciales.

Entre quienes son menos firmes (10,30 \%), se indica que algunas personas docentes se han ido adaptando, pero otras no lo logran del todo - como tampoco lo han logrado las personas estudiantes-, pero es una situación institucional, por ejemplo, lo ilustrado en el siguiente verbatim: "algunos profesores sí, otros no tanto, considero que en general la Universidad de Costa Rica no está preparada para la modalidad virtual, pero ante la emergencia lo ha hecho de buena manera para continuar con el semestre. (sujeto 100, 06 de agosto del 2020).

Otro grupo de 15 personas $(14,8 \%)$ reconoce que algunas de las personas docentes sí contaban con la preparación necesaria, pero resaltan lo importante de su empeño en la vocación, como se ilustra en el siguiente verbatim: "sí, en mi caso, la experiencia fue muy buena... los profesores trataron de dar lo mejor de sí mismos" (sujeto 93, 16 de julio del 2020). Entre estas personas se reconoce que el cuerpo académico encargado de cursos más teóricos lo logran en mayor medida, como lo muestra el siguiente verbatim: "algunos sí, creo que los profesores de cursos teóricos lo logran mejor" (sujeto 27, 29 de junio del 2020); en general buena parte del profesorado logró adaptarse. 
En términos generales, se reconoce el esfuerzo realizado por algunas personas docentes, al tiempo que se critica la falta de empeño de otras con expresiones como:

"... han hecho un gran esfuerzo y han logrado adaptarse a las nuevas condiciones, y en la medida de lo posible han sacado adelante los cursos con buena calidad. Hay otros profesores que no han hecho siquiera el esfuerzo de adaptarse..." (sujeto 47,02 de julio de 2020), "hay profesores que han hecho un excelente trabajo y con una buena preparación, pero en el caso de otros ha sido todo lo contrario" (sujeto 18, 29 de junio de 2020), "todavía les falta, afinar el contenido de presencial a virtual" (sujeto 33, 30 de junio de 2020).

En el otro extremo de la balanza de las percepciones, se encuentran las personas estudiantes que consideran que el profesorado sí estaba preparado para asumir el reto (21,7 \%). Este grupo de estudiantes comprende que a algunas personas docentes pudo haberles costado, pero lo han hecho bien. Se destaca que han tenido muy buenas experiencias en los cursos, que las personas docentes dieron lo mejor de sí, que lo han hecho de muy buena manera y que han utilizado diversas herramientas que les permitieron adaptarse perfectamente a la metodología. Algunas de las expresiones para destacar ese trabajo son: "Considero que todos somos capaces de adaptarnos y que en general los docentes con la preparación que tuvieron lograron con éxito impartir los cursos, aunque siempre hay espacio para mejorar" (sujeto 64, 04 de julio de 2020); "el esfuerzo se nota y es gracias a esto que los cursos se han logrado desenvolver sin grandes contratiempos” (sujeto 5, 28 de junio de 2020); "en realidad sacaron la tarea en tiempo récord, y lograron metas que ni ellos mismos creían haber podido hacer" (sujeto 84, 08 de julio de 2020).

Ahora bien, no solo el cuerpo docente se ha visto en dificultades en esta modalidad. Las personas estudiantes manifestaron sentirse abrumadas y con problemas para desarrollar las actividades propuestas por el profesorado, por ejemplo, los trabajos en grupo. Una opinión particularmente fuerte percibió que:

Algunos profesores se abusaron con la cantidad de trabajos por hacer, considero que estar en mi casa no justifica que tenga más tiempo, al contrario, creo que hay que tomar en cuenta que estar en frente de una computadora durante horas es sumamente agotador para la vista y para cuerpo en general (sujeto 29, 30 de junio de 2020).

De este caso se destaca la importancia de medir el volumen de trabajo asignado y de tomar en cuenta la salud mental de todas las partes involucradas en el proceso.

Al haber vivido la experiencia de pasar súbitamente del aprendizaje presencial al remoto, el estudiantado se posicionó respecto a la naturaleza de los cursos y si estos se adaptan o no a la modalidad virtual.

Según las percepciones del estudiantado, en primer lugar, se puede observar en la Tabla 3 que los 10 cursos que, en su mayoría, se adaptan completamente a la virtualidad son:

TABLA 3

Los 10 cursos que mayoritariamente se adaptan completamente a la virtualidad.

\begin{tabular}{ll}
\hline Curso & Porcentaje \\
\hline 1. Expresión escrita en Francés I & $55,40 \%$ \\
2. Literatura Clásica para Lenguas Modernas & $51,50 \%$ \\
3. Historia y Literatura II & $49,50 \%$ \\
4. Expresión Escrita en Francés II & $47,50 \%$ \\
5. Expresión Escrita en Francés III & $47,50 \%$ \\
6. Historia y Literatura I & $47,50 \%$ \\
7. Gramática Española para Lenguas Modernas & $46,50 \%$ \\
8. Literatura Francesa I & $46,50 \%$ \\
9. Gramática Francesa I & $45,50 \%$ \\
10. Literatura Francesa II & $43,60 \%$ \\
\hline
\end{tabular}

Fuente: elaboración propia.

En segundo lugar, en la Tabla 4 se muestran aquellos cursos que serían aptos para ser trabajados de manera bimodal, para los cuales las 10 opciones con mayoría de respuestas favorables son: 
TABLA 4

Los 10 cursos que mayoritariamente serían aptos para ser trabajados de manera bimodal.

\begin{tabular}{lll}
\hline Curso & Porcentaje \\
\hline 1. & Francés Integrado I & $46,50 \%$ \\
2. & Expresión Oral I & $46,50 \%$ \\
3. Gramática Francesa I & $45,50 \%$ \\
4. & Francés Integrado II & $43,60 \%$ \\
5. Gramática Española para Lenguas Modernas & $42,60 \%$ \\
6. & Expresión Oral II & $42,60 \%$ \\
7. Expresión Oral III & $40,60 \%$ \\
8. Gramática Francesa II & $39,60 \%$ \\
9. Gramática Francesa III & $38,60 \%$ \\
10. & Expresión Oral IV & $37,60 \%$ \\
\hline
\end{tabular}

Fuente: elaboración propia.

En tercer lugar, en la Tabla 5 se destacan los 10 cursos que, según la mayoría del estudiantado, solo deben ser impartidos de manera presencial:

TABLA 5

Los 10 cursos que mayoritariamente solo deben ser impartidos en manera presencial.

\begin{tabular}{lll}
\hline Curso & Porcentaje \\
\hline 1. & Expresión Oral I & $60,40 \%$ \\
2. & Francés Integrado I & $56,40 \%$ \\
3. & Francés Integrado II & $46,50 \%$ \\
4. & Expresión Oral II & $46,50 \%$ \\
5. & Expresión Oral III & $30,70 \%$ \\
6. & Expresión Oral IV & $27,70 \%$ \\
7. & Conversación y Composición II & $21,80 \%$ \\
8. & Conversación y Composición I & $20,80 \%$ \\
9. Traducción I & $14,90 \%$ \\
10. & Lengua y Traducción II & $13,90 \%$ \\
\hline
\end{tabular}

Fuente: elaboración propia.

Una vez destacados los resultados obtenidos de las consultas realizadas, los cuales resaltan tanto aspectos positivos como negativos de la modalidad de enseñanza virtual, la preparación del cuerpo docente y la naturaleza de los cursos, se pasa a reflexionar al respecto.

\section{Discusión DE LOS RESUltados}

Al cabo del análisis de los resultados, se puede constatar que las percepciones de la población estudiantil, de cara a la adaptación del aprendizaje del francés como lengua extranjera, fueron variadas.

De la Figura 3 se desprende que, pese al espíritu crítico del estudiantado, una mayoría considera que el personal docente sí está preparado, independientemente de la variable que se analice. Entre quienes perciben que las personas docentes no están preparadas para asumir la virtualidad en sus cursos, prevalece la cantidad de respuestas de mujeres sobre hombres, de estudiantado matriculado en bloque completo sobre el que no lo está, y de las personas empadronadas en el Bachillerato en Francés; sin embargo, debe tenerse en cuenta que la cantidad de respuestas en las 3 opciones por variables es mayor que su contraparte, con lo cual se intuye que no hay una verdadera incidencia por variable.

Si bien es cierto que se trató de un cambio que se tuvo que hacer de manera repentina y agilizada, dentro del proceso se hallaron características positivas para el proceso de aprendizaje del estudiantado, las cuales, 
no necesariamente, habían sido valoradas antes. Así las cosas, las observaciones hechas por el estudiantado en las respuestas abiertas del cuestionario permiten no solamente identificar áreas de mejora, sino también abrir la posibilidad a nuevas modalidades de enseñanza que se pueden instaurar en adelante en la formación que ofrece la carrera.

Maican y Cocorada (2021), Pastran et al. (2020), Rechidi et al. (2020) y Sánchez (2020) encontraron que la flexibilidad y la accesibilidad de los materiales eran algunas de las ventajas que tenían las TICS durante el contexto de la pandemia, ya que el estudiantado tenía a su disposición los materiales del curso y, además, tenía la posibilidad de entregar sus trabajos o sus tareas en un lapso mucho mayor que en la modalidad presencial. Llama la atención que, en el caso del contexto de esta investigación, la ventaja que fue mencionada por un gran número de estudiantes es el ahorro de tiempo que no ha sido identificado en otras investigaciones. Esto se podría explicar por la situación vial de Costa Rica, ya que, cuando había clases presenciales, el estudiantado tomaba muchas horas para regresar a sus hogares, a pesar de que la distancia era muy corta. Entonces, al pasar los cursos a la virtualidad, el ahorro de tiempo es bastante ventajoso, puesto que, en algunos casos, la duración de un trayecto a la universidad ida y vuelta es de cuatro horas diarias debido a la congestión vehicular.

De igual manera, como observación general con respecto a los inconvenientes del aprendizaje en modalidad virtual, los problemas de conexión y la ausencia de interacción fueron los factores más mencionados por el estudiantado. Esta situación también estaba reflejada en otros estudios, como el de Maican y Cocorada (2021) y de Rechidi et al. (2020). El tiempo de clases por Zoom no permitía tener una interacción frecuente entre el profesorado y el estudiantado o de este con sus pares. Además, la dificultad para el profesorado de responder inmediatamente a las preguntas del estudiantado hacía que las interacciones no fueran tan fluidas, sino que el estudiantado tenía que esperar un cierto lapso para recibir la respuesta.

En cuanto a la conexión de Internet, este fue un problema frecuente en las clases, ya que no todo el estudiantado tenía buena conexión y en casi todas las ocasiones tenían las cámaras apagadas. En Costa Rica, según la Superintendencia de Telecomunicaciones (SUTEL), en su informe de calidad de redes móviles de 2019, se da un porcentaje de área de cobertura a nivel nacional superior o igual a un $90 \%$ para las tecnologías 2G, 3G y 4G. Sin embargo, la calidad de conexión de cada habitante depende de variables específicas como el operador que sirve su región geográfica, el tipo de servicio contratado, el tipo de equipos con que cuenta para conectarse, entre otras. En consideración con lo anterior, y en vista de que el cuestionario fue respondido de manera anónima por las 101 personas participantes y no se les preguntó sobre el lugar de residencia, no es posible comprobar de manera fehaciente sus condiciones de conexión, el nivel de cobertura para cada quien, ni si esta se desagrega de acuerdo con los niveles de vulnerabilidad del estudiantado. Así las cosas, en correspondencia con las preguntas de investigación y la metodología planteadas en la investigación, se evidencia en el presente estudio las percepciones expresadas por las personas participantes.

Esto ocasionaba una falta de interacción durante la clase, ya que el profesorado no podía observar al estudiantado ni sabía si este estaba presente durante toda la clase. Esta misma situación fue identificada por Maican y Coconada (2021), ya que, al haber ausencia de cámaras encendidas por parte del estudiantado, era difícil establecer una interacción fluida entre pares o con el profesorado.

Si bien es cierto que la virtualidad ayudó a mantener los cursos en la universidad y traer ciertas ventajas al proceso de aprendizaje, es importante mencionar que existe todavía una serie de inconvenientes que se deben trabajar para propiciar el aprendizaje más adecuado con el uso de las TICS.

Otro aspecto que señala el estudiantado es el exceso de tiempo delante de la computadora. Este aspecto coincide también con otras investigaciones, como las de Chao (2014) y Contreras (2008), en las cuales se menciona que esto podría generar ansiedad en el estudiantado. De esta manera, es importante equilibrar el tiempo de clases en la virtualidad para que el estudiantado no se sienta cansado o pierda interés cuando las clases son excesivamente largas por Zoom.

Al hablar de la experiencia vivida por las personas estudiantes durante esta transición de lo presencial a lo virtual, se puede afirmar que, dependiendo del año que cursaba el estudiantado, así la vivieron. Al tomar 
en cuenta que, al momento de realizar la matrícula, a nivel institucional no se pensaba que el semestre iba a ser completamente virtual, la mayoría de las personas que respondieron al cuestionario afirmaron llevar el bloque de estudios completo. Esto, sin lugar a duda, tuvo un impacto directo en cómo la población estudiantil afrontó el cambio a la virtualidad. Aquellas personas que seguían cursos de segundo a cuarto año de carrera pudieron expresar su parecer al contrastar su experiencia previa en presencialidad con la nueva virtual. En el caso de estudiantes de primer ingreso, esta experiencia se convirtió en el primer contacto con la vida universitaria.

Si bien es cierto, en un principio el estudiantado calificó de retador y estresante este proceso, 32,6 \% del estudiantado señala que una vez comenzado el ciclo lectivo encontraron la manera de organizar mejor su tiempo y que fue una buena ocasión para utilizar aplicaciones y plataformas en línea; sin embargo, hacen un llamado a no abusar en la cantidad que se utilizan por clase o curso.

Otra observación realizada por las personas participantes es que aún hay oportunidad para mejorar y afinar cómo se imparten los cursos, sobre todo en caso de seguirse ofertando de manera virtual, pues mencionan que se debe hacer un balance en la cantidad de trabajo que se asigna por materia, así como la cantidad de horas por lección, tanto sincrónica como asincrónica. También se resalta la migración oportuna del material que se utiliza en los cursos y lograr que este también sea ofrecido o bien adaptado a la manera digital.

Es así, que, de manera global, como parte de las ventajas halladas, se apunta a que esta experiencia permitió a las personas docentes y estudiantes a ponerse a prueba y a realizar los ajustes necesarios para salir adelante y llevar a cabo el semestre. En el caso de la población estudiantil que aprende una lengua extranjera en modalidad virtual, resultó ser particularmente evidente que se adaptó a ser más autónoma de cara a su proceso de enseñanza-aprendizaje.

Para el éxito del uso de las TICS en clase es necesario que el estudiantado también sea autónomo en su proceso de aprendizaje (Belloch, 2012; Burbat, 2016; Chao, 2014; Contreras, 2008; Durán et al. 2015; Gavarri, 2016; Rodríguez, 2016) y que haya una interacción constante entre el profesorado y el estudiantado (Moreira-Segura y Delgadillo-Espinoza, 2015). Por eso, según las experiencias del estudiantado participante en esta investigación, al principio fue estresante y difícil, pero, conforme cada quien pudo adaptarse a esta nueva modalidad de aprendizaje al adquirir una mayor autonomía en su proceso, se percibió esta experiencia como útil y positiva para poder continuar con los estudios.

Con respecto a la última pregunta de investigación, esta se refiere a describir la percepción que tiene el estudiantado sobre la preparación y adaptación del personal docente a la virtualidad durante el contexto de la pandemia del COVID-19.

La percepción general del estudiantado sobre la experiencia de haber llevado los cursos de carrera virtualmente está en gran parte dividida, ya que, si bien es cierto que fue estresante y cansada, se señala que no todos los cursos se adaptan a la modalidad virtual ni todo el personal docente estaba preparado para impartirlos. En la percepción del estudiantado sobre la preparación y adaptación del personal docente durante el contexto en el que se desarrollaron los cursos, se rescata el profesionalismo de muchas de las personas docentes, el esfuerzo por lograr ofrecer los cursos y por generar una experiencia agradable, buena e interesante. Las personas que respondieron al cuestionario también se centraron en el hecho de que la experiencia de esta modalidad dependió, en gran medida, de la persona docente de cada curso.

Por consiguiente, cabe resaltar que no es posible valorizar la experiencia de aprendizaje únicamente en función de la preparación de la persona docente, su empeño o las estrategias aplicadas, ya que una buena parte de ella depende de la naturaleza de los cursos.

Tal como lo demuestra la variación de respuestas en las que un mismo curso está considerado dentro de los que se adaptan totalmente a la virtualidad y de los que lo hacen de manera parcial o, incluso, dentro de los no pueden ser adaptados y deben ser impartidos de manera presencial, hay quienes destacan la falta de uniformidad en la manera de impartir los cursos. Una persona expresó "Sinceramente los profesores asignados para mis cursos han sabido responder a esta situación, pero aún quedan aspectos por mejorar 
para que haya una unanimidad y que todos los cursos sean trabajados, abordados y evaluados de una misma manera." (sujeto 45, 02 de julio del 2020), y otra indicó:

Creo que sí, mis profes demostraron que estaban muy preparados e interesados para que el proceso de enseñanza fuera provechoso, pero tal y como ellos lo expresaban, la interacción es fundamental para poder avanzar de la forma correcta. La interacción que tuvimos fue una buena respuesta a la situación actual, pero creo que estos tipos de cursos no se deberían impartir virtualmente si la modalidad presencial es posible porque esa es la interacción necesaria para ir más a fondo. (sujeto 82, 07 de agosto de 2020).

Esta última idea parece ser compartida por más personas, por ejemplo, otra persona estudiante declaró que

La universidad cuenta con docentes altamente capacitados para impartir cursos en diferentes modalidades. En lenguas es importante que los cursos sean sincrónicos porque la interacción, en el aprendizaje de idiomas, debe ser mayor que en otros cursos. Si se dan situaciones complicadas, los docentes tienen la capacidad para realizar correcciones, escuchar sugerencias y buscar ayuda en las instancias universitarias según sea el caso. (sujeto 92, 14 de julio de 2020).

El mayor reto destacado por el estudiantado radica en encontrar el equilibrio entre el trabajo sincrónico y asincrónico, entre el diseño instruccional para cursos en línea y el momento propiamente de impartir las lecciones. Ante esto, la sugerencia se centra en aprender y hacer un mayor uso de las herramientas digitales.

Si bien es cierto que no existen muchas investigaciones que documentan la percepción del estudiantado en lenguas extranjeras sobre las experiencias que han tenido en los cursos durante el contexto de la pandemia, las investigaciones sobre las TICS coinciden en que, para aprovechar al máximo los beneficios de estas herramientas, el personal docente debe estar preparado y capacitado (Ait-Dahmane, 2011; Hocine, 2011). En el caso del personal docente, la migración a la virtualidad de los cursos en el primer semestre de 2020 fue algo repentino, por lo cual no hubo suficiente tiempo para capacitarse y prepararse para estos nuevos desafíos, sino que la mayoría del personal docente tuvo que aprender el uso de estas nuevas herramientas durante el desarrollo del semestre. Por eso, en esta investigación, las experiencias vividas por la población estudiantil fueron divididas, ya que hubo una parte de estudiantes que tuvo una vivencia más positiva que otra.

\section{Conclusiones}

Como consecuencia de la aparición de la pandemia COVID-19, la manera de relacionarse entre las personas varió, lo que generó una necesidad de adaptación en todos los ámbitos, particularmente el sistema educativo. Las instituciones implementaron nuevos modelos de enseñanza con el uso de herramientas tecnológicas para seguir impartiendo sus lecciones, lo cual modificó tanto la metodología como la experiencia de aprendizaje del estudiantado. Como lo menciona Burbat (2015), dicha modificación, bien que innovadora, en la metodología desencadena una relectura de los roles que tradicionalmente se han ejercido en la docencia; así se pudo constar gracias a los insumos extraídos del cuestionario aplicado a las personas estudiantes.

En esta investigación, se trataron las siguientes preguntas de investigación: las ventajas y desventajas del aprendizaje de una lengua extranjera en modalidad virtual; la experiencia del estudiantado sobre los cursos de francés, y la percepción del estudiantado sobre la preparación y adaptación del personal docente a la virtualidad.

Es así como un cuestionario aplicado en línea al estudiantado, en el contexto de aprendizaje del francés como lengua extranjera en modalidad virtual durante pandemia por COVID-19, permitió recolectar información que indica que las ventajas del aprendizaje del francés en modalidad virtual son: ahorro de tiempo de traslado y de dinero, flexibilidad para la realización de los trabajos, comodidad de recibir clases en la casa, así como la organización en su propio ritmo de trabajo y de su aprendizaje, en términos de manejo de estrés y coordinación con sus vidas, tanto en el ámbito personal como laboral. Por otra parte, las desventajas señaladas por el estudiantado mediante la información brindada, también dan cuenta de los inconvenientes de esta modalidad de aprendizaje, que van desde los problemas de conexión a Internet hasta 
la ausencia de interacción con el profesorado y con pares, lo que afectó la retroalimentación y el desarrollo de sus competencias comunicativas, particularmente a nivel oral.

De manera general, las percepciones de la población estudiantil sobre la adaptación del aprendizaje del francés como lengua extranjera a la virtualidad demuestran que esta experiencia permitió a docentes y a estudiantes ponerse a prueba y realizar los ajustes necesarios para salir adelante y llevar a cabo el semestre. En el caso de la población estudiantil, resultó ser particularmente evidente que se adaptó a ser más autónoma de cara a su proceso de enseñanza-aprendizaje. Este aspecto es también señalado por Bachelor (2019), quien apunta que, en el contexto de un curso virtual, la persona estudiante debe ser más independiente y contar con mayor motivación para aprender un idioma extranjero. Por lo tanto, en este nuevo contexto, es necesario otorgar una participación más activa al estudiantado, mientras que la persona docente debe tener un rol más mediador y de guía durante este proceso de enseñanza-aprendizaje.

Por último, las personas estudiantes fueron críticas con respecto a la preparación, la adaptación y el desempeño de las personas docentes a la virtualidad durante el contexto de la pandemia del COVID-19, pues reconocen que existen grandes espacios de mejora a pesar de su esfuerzo y voluntad de adaptación, ya que algunas personas docentes se han ido adaptando, pero otras no lo logran del todo - como tampoco lo ha logrado el estudiantado-. Esta es una situación global, ya que, en general, las instituciones no estaban preparadas para una migración repentina hacia la virtualidad. Así como lo señala Kem-mekah (2020), los cambios realizados en el contexto educativo a causa de la emergencia global por el coronavirus no son simplemente una opción, sino una necesidad, y dichos cambios fueron realizados a partir de los recursos con los que ya se contaba.

\section{Recomendaciones}

En vista de que esta investigación compila las percepciones luego de los primeros meses de enseñanza del francés desde la virtualidad, se hace evidente la utilidad de realizar un nuevo estudio algunos meses después, que permita identificar si las debilidades, en términos de formación en TICS por parte del cuerpo docente, y de adaptación y, tanto por parte de docentes como de estudiantes, han sido superadas. También sería interesante realizar una investigación longitudinal con esta misma población de estudiantes, para conocer si ha habido cambios en su experiencia con respecto a la virtualidad en los cursos. Más allá, es importante identificar si las metodologías aplicadas han sido de utilidad en términos de cumplimiento de objetivos de formación universitaria, y si estas se asemejan o difieren de otras instituciones a nivel regional. Ya lo señala Belloch (2012), cuando afirma que gracias a los avances tecnológicos en el área de la educación hay más cabida para la innovación y así la posibilidad de proponer una relectura de los procesos de enseñanza y aprendizaje, como también lograr una actualización continua de las personas profesionales en educación.

El contexto mundial anuncia que la virtualidad llegó para quedarse, y que se debe estudiar para sacar el mayor provecho de ella. Por lo tanto, algunas recomendaciones que se puede realizar sobre esta adaptación son: capacitar mejor al personal docente sobre la utilización de las TICS en la enseñanza de una lengua en la virtualidad, compartir experiencias entre el personal docente para fomentar las buenas prácticas de enseñanza en la virtualidad, experimentar con nuevas formas y herramientas para adaptarse a este nuevo contexto, entre otras.

\section{REFERENCIAS BIBLIOGRÁFICAS}

Ait-Dahmane, K. (2011). L'impact des TICE sur l'enseignement/l'apprentissage de la langue française dans le supérieur: quels besoins de formation pour quelle pédagogie? Synergies Algérie, 12, 227-231. https://gerflint.fr /Base/Algerie12/karima_ait-dahmane.pdf

Al-Assaf, Y. (2008). L'internet au service de l'enseignement de la langue française. Adab Al-Rafidayn, 49, 125-146. h ttps://www.iasj.net/iasj/download/d6592eb60e326f21 
Kuok Wa Chao Chao, et al. Educación universitaria en tiempos de pandemia: el caso del estudiantado...

Bachelor, J. (2019). El aula presencial, semipresencial, virtual e invertida: un estudio comparativo de métodos didácticos en la enseñanza de L2. Revista Educación, 43(2), 1-20. https://doi.org/10.15517/revedu.v43i2.34014

Beauné, A. (2011). Quelles utilisations des TICE pour l'apprentissage du français langue étrangère au niveau A1.1? [Tesis de Maestría], Universidad París-Descartes. https://adjectif.net/IMG/pdf/M2.pdf

Belloch, C. (2012). Las Tecnologías de la Información y Comunicación en el aprendizaje. Material docente [on-line]. Departamento de Métodos de Investigación y Diagnóstico en Educación. Universidad de Valencia. http://ww w.uv.es/bellochc/pedagogia/EVA1.pdf

Burbat, R. (2016). El aprendizaje autónomo y las TIC en la enseñanza de una lengua extranjera: Progreso o Retroceso? Porta Linguarum, 26, 37-51. https://www.ugr.es/ portalin/articulos/PL_numero26/ART3_Ruth\%20Burba t.pdf

Chao, K. W. (2014). Estrategias didácticas mediadas con TIC en un curso de expresión oral francesa. Actualidades Investigativas en Educación, 14(2), 1-30. https://doi.org/10.15517/AIE.V14I2.14801

Cevallos, K. G., Palma, M. A., Cevallos, K. M. y Baquezea, G. V. (2020). Enseñanza del inglés como lengua extranjera (EFL) en el desarrollo de la destreza speaking a través de clases virtuales en la educación superior. Revista Cognosis, 5, 167-177. https://doi.org/10.33936/cognosis.v5i0.2785

Contreras, N. (2008). La enseñanza-aprendizaje de lenguas extranjeras y las TICs: el caso de español como lengua extranjera (ELE). Iniciación a la Investigación, 3, 1-7. https://revistaselectronicas.ujaen.es/index.php/ininv/art icle/view/233

Creswell, J. W. (2009). Research design: Qualitative, quantitative and mixed methods approaches (3ra ed.). Sage Publications.

Durán, R. (2015). La Educación Virtual Universitaria como medio para mejorar las competencias genéricas y los aprendizajes a través de buenas prácticas docentes. [Tesis Doctoral], Universidad Politécnica de Cataluña. https ://tdx.cat/handle/10803/397710

Durán, R., Estay-Niculcar, C. y Álvarez, H. (2015). Adoption of good virtual education practices in higher education. Aula Abierta, 43(2), 77-86. https://doi.org/10.17811/rifie.43.2015.77-86

Fortin, M. F. (2010). Fondements et étapes du processus de recherche: Méthodes quantitatives et qualitatives. (2da edición). Chenelière éducation.

Gavarri, S. L. (2016). El aprendizaje de lenguas extranjeras mediado por las TIC: Aprender inglés con Duolingo. Memoria Académica, 7(12), 56-65. http://www.memoria.fahce.unlp.edu.ar/art_revistas/pr.7199/pr.7199.pdf

Gil, N. A. (2019). Ambiente virtual de aprendizaje: beneficios y ventajas para enseñanza del francés como L2. Revista Boletin Redipe, 8(11), 91-99. https://doi.org/10.36260/rbr.v8i11.852

Hocine, N. (2011). Intérêts pédagogiques de l'intégration des TICE dans l'enseignement du FLE: l'utilisation du webblog dans des activités de production écrite. Synergies Algérie, 12, 219-226. https://gerflint.fr/Base/Algerie12/ naima_hocine.pdf

Kem-mekah, O. (2020). Enseñanza en línea durante la crisis del COVID-19 en la educación universitaria camerunesa: logros y desafíos. Ehquidad International Welfare Policies and Social Work Journal, 14, 57-74. https://doi.org/ 10.15257/ehquidad.2020.0012

Lizasoain, A., Ortiz, A. y Becchi, C. (2018). Utilización de una herramienta TIC para la enseñanza del inglés en un contexto rural. Educ. Pesqui., 44, 1-22. https://doi.org/10.1590/s1678-4634201844167454

Maican, M. A. y Cocorada, E. (2021). Online Foreign Language Learning in Higher Education and Its Correlates during the COVID-19 Pandemic. Sustainability, 13, 1-21. https://doi.org/10.3390/su13020781

Martínez, V. (2017). Educación presencial versus educación a distancia. La Cuestión Universitaria, 9, 108-116. http: //polired.upm.es/index.php/lacuestionuniversitaria/article/view/3582

Martínez, J., Julià, A. y Tejedor, S. (2014). Tecnología y pedagogía en las aulas. Gabinete de Comunicación y Educación de la Universidad Autónoma de Barcelona. Editorial Planeta S.A.U.

Moreira-Segura, C. y Delgadillo-Espinoza, B. (2015). La virtualidad en los procesos educativos: reflexiones teóricas sobre su implementación. Tecnología en Marcha, 28 (1), 121-129. https://doi.org/10.18845/tm.v28i1.2196 
Navarro-Hudiel, S. J. (2020). Tendencias en el uso de recursos y herramientas de la tecnología en la educación universitaria ante la pandemia covid-19. Revista Ciencia y Tecnología El Higo, 10 (2), 111-122. https://doi.org /10.5377/elhigo.v10i2.10557

Organización Mundial de la Salud [OMS]. (2020, 11 de marzo). Alocución de apertura del Director General de la OMS en la rueda de prensa sobre la COVID-19 celebrada el 11 de marzo de 2020. Organización Mundial de la Salud. https://www.who.int/es/director-general/speeches/detail/who-director-general-s-opening-remarks-at-t he-media-briefing-on-covid-19---11-march-2020

Otzen, T. y Manterola, C. (2017). Técnicas de muestreo sobre una población a estudio. Int. J. Morphol., 35 (1), 227-232. https://scielo.conicyt.cl/pdf/ijmorphol/v35n1/art37.pdf

Pastran, M., Gil, N. A. y Cervantes, D. (2020). En tiempos de coronavirus: las TIC'S son una buena alternativa para la educación remota. Revista Boletín Redipe, 9(8), 158-165. https://doi.org/10.36260/rbr.v9i8.1048

Pereira, Z. (2011). Los diseños de método mixto en la investigación en educación: Una experiencia concreta. Revista Electrónica Educare, 15(1), 15-29. https://www.redalyc.org/pdf/1941/194118804003.pdf

Rechidi, N., Bennani, H., Nafzaoui, M. A. y Benazzou, L. (2020). L'intégration pédagogique des TIC à l'épreuve de la crise covid-19 : quels enseignements à tirer? Revue internationale du chercheur, 1(2), 274-297. https://doi.or $\mathrm{g} / 10.5281 /$ zenodo.3877605

Rodríguez, N. (2016). ¿Las TIC como mediadoras en la enseñanza-aprendizaje de lenguas extranjeras?. Opción, 32(10), 569-588. https://www.redalyc.org/pdf/310/31048901031.pdf

Ruiz, M. del R. y Aguirre, G. A. (2013). Quehacer docente, TIC y educación virtual o a distancia. Apertura: Revista de Innovación Educativa, 5(2), 108-123. https://www.redalyc.org/pdf/688/68830444010.pdf

Sánchez, A. (2020). Microteaching at home. Formato de la creatividad y el aprendizaje "lúcdico" en la Universidad en tiempos de la COVID-19. Magister, 32(1), 61-65. https://doi.org/10.17811/msg.32.1.2020.62-65

Simons, M. (2010). Perspectiva didáctica sobre el uso de las TIC en clase de ELE. Revista de didáctica español como lengua extranjera, 11, 1-21. https://marcoele.com/descargas/11/simons-tic.pdf

Soto, A. B. (2018). Enseñar literatura en lengua extranjera con herramientas digitales: reflexiones generales desde la didáctica de FLE. Revista de estudios filológicos, 35, 1-24. https://dialnet.unirioja.es/servlet/articulo?codigo=65 15699

Superintendencia de telecomunicaciones (SUTEL). (2019). Informe de principales resultados de la evaluación nacional de la calidad de los servicios móviles. SUTEL.

\section{INFORMACIÓN ADICIONAL}

Cómo citar: Chao-Chao, K. A., Cambronero-Artavia, M. P. y Chacón-Quesada, M. P. (2022). Educación universitaria en tiempos de pandemia: el caso del estudiantado de francés. Revista Educación, 46(1). http:/ /doi.org/10.15517/revedu.v46i1.45343 